Pacific

Journal of

Mathematics

NUMERICAL SEMIGROUPS GENERATED BY INTERVALS P.A. García-SÁnchez and J.C. Rosales 


\title{
NUMERICAL SEMIGROUPS GENERATED BY INTERVALS
}

\author{
P.A. García-SÁnchez and J.C. Rosales
}

We study numerical semigroups generated by intervals and solve the following problems related to such semigroups: the membership problem, give an explicit formula for the Frobenius number, decide whether the semigroup is a complete intersection and/or symmetric, and computation of the cardinality of a (any) minimal presentation of this kind of numerical semigroups.

A numerical semigroup is a finitely generated subsemigroup of the set of nonnegative integers $\mathbb{N}$, such that the group generated by it is the set of all integers $\mathbb{Z}$. In this paper we study the semigroups generated by intervals of nonnegative integers, that is to say, semigroups of the form $S=\langle a, a+$ $1, \ldots, a+x\rangle=\left\{\sum_{i=0}^{x} n_{i}(a+i): n_{i} \in \mathbb{N}\right\} \subseteq \mathbb{N}$. Note that if $x \geq a$, then $S=\{a, a+1, \ldots\}=a+\mathbb{N}=\langle a, a+1, \ldots, 2 a-1\rangle ;$ thus we may assume that $x \leq a-1$. For a semigroup of this kind we solve the following problems:

1) Membership problem. An element $n \in \mathbb{N}$ belongs to $S=\langle a, a+$ $1, \ldots, a+x\rangle$ if and only if $n \bmod a \leq\left\lfloor\frac{n}{a}\right\rfloor x$, where $\left\lfloor\frac{n}{a}\right\rfloor$ is quotient of the integer division of $n$ by $a$, and $n \bmod a$ denotes the remainder of this division, $n-\left\lfloor\frac{n}{a}\right\rfloor a$.

2) Computation of the Frobenius number of the semigroup. The Frobenius number of a numerical semigroup (also known as the conductor of the semigroup) is the greatest integer not belonging to the given semigroup. The Frobenius number of $S=\langle a, a+1, \ldots, a+x\rangle$ is $\left\lceil\frac{a-1}{x}\right\rceil a-1$, where $\lceil q\rceil$ denotes the least integer greater than or equal to $q \in \mathbb{Q}^{+}$.

3) Symmetry of the semigroup. A numerical semigroup $T$ with Frobenius number $C$ is symmetric if and only if for each $z \in \mathbb{Z}$ we have that either $z \in S$ or $C-z \in S$. These kinds of semigroups are specially interesting in Ring Theory as Kunz shows in [7]. The semigroup $S=$ $\langle a, a+1, \ldots, a+x\rangle$ is symmetric if and only if $a \equiv 2 \bmod x$ (here $a \equiv b \bmod c$ denotes the fact $a-b=k c$ for some integer $k$ ).

4) Cardinality of a minimal presentation of $S$. The semigroup $S=\langle a, a+$ $1, \ldots, a+x\rangle$ is isomorphic to $\mathbb{N}^{x+1} / \sigma$, where $\sigma$ is the kernel congruence of the semigroup morphism

$$
\varphi: \mathbb{N}^{x+1} \rightarrow S, \varphi\left(n_{0}, \ldots, n_{x}\right)=\sum_{i=0}^{x} n_{i}(a+i),
$$


that is to say $a \sigma b$ if and only if $\varphi(a)=\varphi(b)$. A minimal presentation of $S$ is a minimal system of generators of the congruence $\sigma$. In this paper we show that the cardinality of a minimal presentation of $S$ is

$$
\frac{x(x-1)}{2}+x-((a-1) \bmod x) .
$$

5) Complete intersection semigroups. A numerical semigroup is a complete intersection if the cardinality of a minimal presentation plus one equals the cardinality of a minimal system of generators of the given semigroup (see [6]). We show that the semigroup $S=\langle a, a+$ $1, \ldots, a+x\rangle$ is a complete intersection if and only if $S$ is $\langle a, a+1\rangle$ or $\langle 2 k, 2 k+1,2 k+2\rangle$.

The point of departure to solve these problems is the following lemma.

Lemma 1. Let $S=\langle a, a+1, \ldots, a+x\rangle$ be a numerical semigroup with $1 \leq x<a$. Then, $n \in S$ if and only if $n=q a+i$ with $q \in \mathbb{N}$ and $i \in\{0, \ldots, q x\}$.

Proof. If $n \in S$ then there exist $n_{0}, \ldots, n_{x} \in \mathbb{N}$ such that $n=\sum_{j=0}^{x} n_{j}(a+j)$. Thus, $n=\left(\sum_{j=0}^{x} n_{j}\right) a+\sum_{j=1}^{x} n_{j} j$. Take $q=\sum_{j=0}^{x} n_{j}$ and $i=\sum_{j=1}^{x} n_{j} j \leq$ $\sum_{j=0}^{x} n_{j} x=q x$.

Now, assume that $n=q a+i$, with $0 \leq i \leq q x$. We distinguish two possible cases:

1) If $i=q x$ then $n=q(a+x) \in S$.

2) If $i=k x+r$, with $0 \leq k<q$ and $0 \leq r \leq x-1$, then $n=q a+k x+r=$ $(q-k-1) a+k(a+x)+a+r \in S$.

From this membership characterization, we can derive the following characterization which is easier to check and it is what we will use later in the paper.

Corollary 2. Let $S=\langle a, a+1, \ldots, a+x\rangle$ be a numerical semigroup with $1 \leq x<a$. Then, $n \in S$ if and only if $(n \bmod a) \leq\left\lfloor\frac{n}{a}\right\rfloor x$.

Proof. If $n \in S$, then using the previous lemma, there exists $q \in \mathbb{N}$ and $0 \leq i \leq q x$ such that $n=q a+i$. Besides, $n=\left\lfloor\frac{n}{a}\right\rfloor a+(n \bmod a)$. Thus, $n \bmod a=q a+i-\left\lfloor\frac{n}{a}\right\rfloor a=\left(q-\left\lfloor\frac{n}{a}\right\rfloor\right) a+i$, and since $q \leq\left\lfloor\frac{n}{a}\right\rfloor$, we get that $n \bmod a \leq i \leq q x \leq\left\lfloor\frac{n}{a}\right\rfloor x$.

Now, assume that $(n \bmod a) \leq\left\lfloor\frac{n}{a}\right\rfloor x$. Since $n=\left\lfloor\frac{n}{a}\right\rfloor a+(n \bmod a)$ and $0 \leq n \bmod a \leq\left\lfloor\frac{n}{a}\right\rfloor x$, applying the previous result, we get that $n \in S$.

For every $n \in S$, the Apéry set (see [1]) associated to $n$ is defined as

$$
\mathrm{S}(n)=\{s \in S: s-n \notin S\} .
$$

Using last result, we can characterize the elements belonging to $\mathrm{S}(a)$. This characterization is going to play an important role in the rest of the paper. 
Corollary 3. Let $S=\langle a, a+1, \ldots, a+x\rangle$ be a numerical semigroup with $1 \leq x<a$ and let $n \geq a$. Then, $n \in \mathrm{S}(a)$ if and only if $(n \bmod a) \in$ $\left\{\left(\left\lfloor\frac{n}{a}\right\rfloor-1\right) x+1, \ldots,\left\lfloor\frac{n}{a}\right\rfloor x\right\}$.

Proof. The element $n \in \mathrm{S}(a)$ if and only if $n \in S$ and $n-a \notin S$. By the previous result this occurs if and only if $(n \bmod a) \leq\left\lfloor\frac{n}{a}\right\rfloor x$ and $((n-$ a) $\bmod a)>\left\lfloor\frac{n-a}{a}\right\rfloor x$. But $(n-a) \bmod a=n \bmod a$ and $\left\lfloor\frac{n-a}{a}\right\rfloor=\left\lfloor\frac{n}{a}\right\rfloor-1$ (note that $n \geq a$ ). Hence, $n \in \mathrm{S}(a)$ if and only if $n \bmod a \in\left\{\left(\left\lfloor\frac{n}{a}\right\rfloor-1\right) x+\right.$ $\left.1, \ldots,\left\lfloor\frac{n}{a}\right\rfloor x\right\}$.

We can explicitly construct $\mathrm{S}(a)$ as the next corollary shows.

Corollary 4. Let $S=\langle a, a+1, \ldots, a+x\rangle$ be a numerical semigroup with $1 \leq x<a$. Then, $\mathrm{S}(a)=\{q a+(q-1) x+r: 1 \leq r \leq x, q \in \mathbb{N}$ and $0 \leq$ $(q-1) x+r<a\}$.

Proof. Take $n=q a+(q-1) x+r$ such that $1 \leq r \leq x, q \in \mathbb{N}$ and $0 \leq$ $(q-1) x+r<a$. Then, $\left\lfloor\frac{n}{a}\right\rfloor=q$ and $n \bmod a=(q-1) x+r=\left(\left\lfloor\frac{n}{a}\right\rfloor-1\right) x+r \in$ $\left\{\left(\left\lfloor\frac{n}{a}\right\rfloor-1\right) x+1, \ldots,\left\lfloor\frac{n}{a}\right\rfloor x\right\}$. Using the previous result, we get that $n \in \mathrm{S}(a)$.

Now, take $n=\left\lfloor\frac{n}{a}\right\rfloor a+(n \bmod a) \in \mathrm{S}(a)$. Then, by the previous corollary, $n \bmod a \in\left\{\left(\left\lfloor\frac{n}{a}\right\rfloor-1\right) x+1, \ldots,\left\lfloor\frac{n}{a}\right\rfloor x\right\}$ and therefore $n \bmod a=\left(\left\lfloor\frac{n}{a}\right\rfloor-1\right) x+r$, with $r \in\{1, \ldots, x\}$. Taking $q=\left\lfloor\frac{n}{a}\right\rfloor$ we are done.

If $S$ is a numerical semigroup then the set $\mathbb{N} \backslash S$ is finite, because the group spanned by $S$ is $\mathbb{Z}$. As we have mentioned before, the maximum of this set is called the Frobenius number of the semigroup, which we denote by $\mathrm{C}(S)$. It is well known (see [2]) that $\mathrm{C}(S)=\max (\mathrm{S}(a))-a$. Thus, if we want to compute $\mathrm{C}(S)$, we have to determine the greatest element in $\mathrm{S}(a)$. This is performed in the next result.

Corollary 5. Let $S=\langle a, a+1, \ldots, a+x\rangle$ be a numerical semigroup with $1 \leq x<a$. Then, $\mathrm{C}(S)=\left\lceil\frac{a-1}{x}\right\rceil a-1$.

Proof. We must determine the maximum of $\mathrm{S}(a)=\{q a+(q-1) x+r: 1 \leq$ $r \leq x, q \in \mathbb{N}$ and $0 \leq(q-1) x+r<a\}$. The maximum is reached when $(q-1) x+r=a-1$. The element $a-1$ is equal to $\left\lfloor\frac{a-1}{x}\right\rfloor x+((a-1) \bmod x)$. Two possibilities arise:

- If $(a-1) \bmod x \neq 0$ then take $(q-1)=\left\lfloor\frac{a-1}{x}\right\rfloor$ and $r=(a-1) \bmod x$. The greatest element in $\mathrm{S}(a)$ is $q a+(q-1) x+r=\left(\left\lfloor\frac{a-1}{x}\right\rfloor+1\right) a+a-1$.

- If $(a-1) \bmod x=0$ then, since $r$ must be in $\{1, \ldots, x\}$, write $a-1$ as $a-1=\left(\left\lfloor\frac{a-1}{x}\right\rfloor-1\right) x+x$. Take $q-1=\left\lfloor\frac{a-1}{x}\right\rfloor-1$ and $r=x$. In this case, the greatest element in $\mathrm{S}(a)$ is $q a+(q-1) x+r=q a+(q-1) x+x=$ $\left\lfloor\frac{a-1}{x}\right\rfloor a+a-1$.

Both cases are represented by $\left\lceil\frac{a-1}{x}\right\rceil a+a-1$, which is the greatest element in $\mathrm{S}(a)$. 
Note that this implies that for the numerical semigroups generated by intervals there exists an explicit formula to compute the Frobenius number of the semigroup. It seems that there is no known formula for the general case. Nevertheless, for some specific cases there exists an explicit formula. For instance, in [4], a formula for the Frobenius number of numerical semigroups generated by up to three elements and of symmetric numerical semigroups generated by up to four elements is given (see also [5] for more references).

Another characterization of symmetric numerical semigroups is the following (see $[3]$ ). The numerical semigroup $S$ is symmetric if the greatest element, $w$, of $\mathrm{S}(a)$ satisfies the condition that for every $s \in \mathrm{S}(a)$, the element $w-s$ is in $S$. We use this result to give a characterization of the numerical semigroups generated by intervals that are symmetric.

Theorem 6. Let $S=\langle a, a+1, \ldots, a+x\rangle \neq \mathbb{N}$ be a numerical semigroup with $1 \leq x<a$. Then, $S$ is symmetric if and only if $a \equiv 2 \bmod x$.

Proof. We can assume that $x \geq 2$, since if $x=1$ then $S$ is generated by two relatively prime elements and in this case it is well known that $S$ is symmetric (see for instance [5]).

Let $w$ be the greatest element in $\mathrm{S}(a)$. We already know that $w=q a+$ $(q-1) x+r$ with $(q-1) x+r=a-1$.

If $S$ is symmetric, since $1 \notin S$, then $q a+(q-1) x+r-1$ cannot be in $\mathrm{S}(a)$. Hence, $q a+(q-1) x+r-1 \in S \backslash \mathrm{S}(a)$, which means that $n=(q-1) a+(q-$ $1) x+r-1=q a+(q-1) x+r-1-a \in S$. Since $n \bmod a=(q-1) x+r-1$, $\left\lfloor\frac{n}{a}\right\rfloor=q-1$ and $n \bmod a$ must be less than or equal to $\left\lfloor\frac{n}{a}\right\rfloor x$, we get that $r-1$ must be zero. Hence, $a-1=(q-1) x+1$, which means that $a \equiv 2 \bmod x$.

If $a \equiv 2 \bmod x$, then $(a-1) \equiv 1 \bmod x$, and therefore $(a-1) \bmod x=1$, which from the first case in the proof of the last corollary implies that $r=1$. Consequently, $w=q a+(q-1) x+1$. Take $0 \neq m=k a+(k-1) x+i \in \mathrm{S}(a)$, that is to say $0 \neq k \in \mathbb{N}, 0 \leq(k-1) x+i<a$ and $i \in\{1, \ldots, x\}$. Then, $w-m=(q-k) a+(q-k-1) x+(x-i+1)$. Note that $x-i+1 \in\{1, \ldots, x\}$, $q-k \in \mathbb{N}$ and $0 \leq(q-k-1) x+(x-i+1)=(q-k) x+1-i<a$. Hence, $w-m \in \mathrm{S}(a) \subset S$ for all $m \in \mathrm{S}(a)$ and this means that $S$ is symmetric.

The rest of the paper is devoted to computing the cardinality of a minimal presentation of the numerical semigroup $S=\langle a, a+1, \ldots, a+x\rangle$ with $1 \leq x<a$.

It can be shown (see [6] for example) that for a numerical semigroup the cardinality of a set of generators of $\sigma$, the kernel congruence of $\varphi$, is greater than or equal to the number of generators of the semigroup minus one (in our case, this amount is $x$ ). When this lower bound is reached, the semigroup is a complete intersection. These semigroups are always symmetric.

In [8], the first author gives an algorithm to compute a system of generators, $\rho$, for $\sigma$ with minimal cardinality. From the results given in that paper, it is determined that the concepts of system of generators for $\sigma$ with 
minimal cardinality and minimal system (with respect to the inclusion) of generators of $\sigma$ coincide. Next, we give a sketch of this construction, which is needed to count the elements in a minimal presentation of $S$.

For every $n \in S$, we define the graph $\mathrm{G}_{n}=\left(\mathrm{V}_{n}, \mathrm{E}_{n}\right)$, as

$$
\begin{gathered}
\mathrm{V}_{n}=\{a+i \in\{a, \ldots, a+x\}: n-(a+i) \in S\}, \\
\mathrm{E}_{n}=\{[a+i, a+j]: n-((a+i)+(a+j)) \in S, i \neq j \in\{0, \ldots, x\}\} .
\end{gathered}
$$

We define $\rho_{n}$ as:

1) If $\mathrm{G}_{n}$ is not connected and $\mathrm{G}_{n}^{1}=\left(\mathrm{V}_{n}^{1}, \mathrm{E}_{n}^{1}\right), \ldots, \mathrm{G}_{n}^{r}=\left(\mathrm{V}_{n}^{r}, \mathrm{E}_{n}^{r}\right)$ are its connected components, then for every $1 \leq i \leq r$ we select an element $\alpha_{i}=\left(n_{0_{i}}, \ldots, n_{x_{i}}\right) \in \mathbb{N}^{x+1} \backslash\{0\}$ such that $\varphi\left(\alpha_{i}\right)=n$ and $n_{k_{i}}=0$ for all $a+k_{i} \notin \mathrm{V}_{n}^{i}$. Define $\rho_{n}=\left\{\left(\alpha_{1}, \alpha_{2}\right),\left(\alpha_{1}, \alpha_{3}\right), \ldots,\left(\alpha_{1}, \alpha_{r}\right)\right\}$.

2) If $\mathrm{G}_{n}$ is connected, we define $\rho_{n}=\emptyset$.

The set $\rho=\bigcup_{n \in S} \rho_{n}$ is a system of generators for $\sigma$ with minimal cardinality (see [8]). Thus, we must look for the elements $n \in S$ such that $\mathrm{G}_{n}$ is not connected.

Example 7. Let $S=\langle 6,7,8\rangle$. The vertices of $\mathrm{G}_{14}$ are $\{6,7,8\}$ and the only edge of $\mathrm{G}_{14}$ is $[6,8]$. Thus $\mathrm{G}_{14}$ has two connected components: One is the vertex 7 and the other is the edge $[6,8]$. The elements $(0,2,0)$ and $(1,0,1)$ are in $\mathbb{N}^{3}$ and verify that $\varphi(0,2,0)=\varphi(1,0,1)=14$ (observe that the first and last coordinates of $(0,2,0)$ are zero and that the second coordinate of $(1,0,1)$ is zero). Thus $\rho_{14}=\{((0,2,0),(1,0,1))\}$, meaning that $2 \times 7=6+8$ is a relation on $S$.

Theorem 8. Let $S=\langle a, a+1, \ldots, a+x\rangle$ be a numerical semigroup with $1 \leq x<a$ and $\rho$ as before. Then,

$$
\# \rho=\frac{x(x-1)}{2}+x-((a-1) \bmod x) .
$$

Proof. From the proof of Lemma 1, it is derived that if $n \in S$ then $n$ can be expressed in one of the following ways:

- $n=k a+l(a+x)$ for some nonnegative integers $k, l$.

- $n=k a+l(a+x)+(a+i)$ for some nonnegative integers $k, l$ and $i \in\{1, \ldots, x-1\}$.

Hence, if $\mathrm{G}_{n}$ is not connected, then the set $\{a, a+x\} \cap \mathrm{V}_{n}$ is not empty. In the construction of $\rho$ we take $\mathrm{G}_{n}^{1}$ to be the connected component containing $a$, if $a \in \mathrm{V}_{n}$. If $a$ is not a vertex of $\mathrm{G}_{n}$ then we take $\mathrm{G}_{n}^{1}$ to be the connected component containing $a+x$ (which must be in $\mathrm{V}_{n}$ ). We are going to count the elements in $\rho$ which come from the fact that $a+i, 1 \leq i<x$ is neither in the connected component containing $a$ nor in the connected component containing $a+x$ (if $a$ or $a+x$ is not in $\mathrm{V}_{n}$ this is translated to the fact that $a+i$ is not in $\mathrm{V}_{n}^{1}$ ). Next, we will count the elements in $\rho$ which arise when 
$a$ and $a+x$ are in different connected components (that is to say, $a+x$ not in $\mathrm{V}_{n}^{1}$ ). With this, we count all the elements belonging to $\rho$.

- First, let us assume that $\mathrm{G}_{n}$ is not connected and there is $a+i \in$ $\mathrm{V}_{n}$ such that $a+i$ is not in the connected component(s) containing $\{a, a+x\} \cap \mathrm{V}_{n}$. Hence, $n-(a+i+a) \notin S$ and $n-(a+i+a+x) \notin S$, which implies that $n=w+(a+i)$ with $0 \neq w \in \mathrm{S}(a) \cap \mathrm{S}(a+x)$. It is easy to check, from the description of $\mathrm{S}(a)$ given in Corollary 4 , that $\mathrm{S}(a) \cap \mathrm{S}(a+x)=\{0, a+1, \ldots, a+x-1\}$. Thus, there exists $j \in\{1, \ldots, x-1\}$ such that $n=(a+j)+(a+i)$. Note that the reverse is also true: If $n=(a+i)+(a+j)$ with $i, j \in\{1, \ldots, x-1\}$, then the elements $a$ and $a+i$ are not connected in $\mathrm{G}_{n}$ and the same holds for $a+x$ and $a+i$. This means that every expression of the form $n=(a+i)+(a+j)$ with $1 \leq i, j \leq x-1$ yields a new element in $\rho_{n}$ (the element $\left(\alpha_{1}, \alpha_{t}\right)$, where $\mathrm{G}_{n}^{t}$ is the connected component of $\mathrm{G}_{n}$ containing $\left.a+i\right)$. This implies that from these graphs we get as many elements in $\rho$ as pairs $(i, j)$ with $1 \leq i, j \leq x-1$. This amount is $x(x-1) / 2$.

- Now, let us count the elements in $\rho$ coming from non-connected graphs $\mathrm{G}_{n}$ such that $a$ and $a+x$ are in different connected components. Since $a$ and $a+x$ are in different connected components of $\mathrm{G}_{n}$, the element $n$ can be expressed as $n=w+(a+x)$, where $w \in \mathrm{S}(a)$. Note also that $n \notin \mathrm{S}(a)$. Thus, we must find the elements $w$ in $\mathrm{S}(a)$ such that $w+(a+x)-a=w+x \in S$. By the proof of Corollary 5, depending on $r=(a-1) \bmod x$, the maximum element in $\mathrm{S}(a)$ is $q a+(q-1) x+r$, if $r \neq 0$, or $q a+(q-1) x+x$, if $r=0$, where $q=\left\lceil\frac{a-1}{x}\right\rceil$.

1) If $r \neq 0$, then $q=\left\lfloor\frac{a-1}{x}\right\rfloor+1 \geq 2$. Let us show that the element $w=k a+(k-1) x+i \in \mathrm{S}(a) \quad(1 \leq i \leq x$ and $0 \leq(k-1) x+i<$ a) satisfies that $w+x$ is not in $S$ when $k \leq q-2$. Note that $(k-1) x+i+x \leq(k+1) x \leq(q-1) x=\left\lfloor\frac{a-1}{x}\right\rfloor x \leq a-1<a$. Thus, $(w+x) \bmod a=k x+i$ and $\left\lfloor\frac{w+x}{a}\right\rfloor x=k x$. Since $i \geq 1$, by Corollary $2, w+x \notin S$.

Let us show that $w=(q-1) a+(q-2) x+i \in \mathrm{S}(a)$ verifies that $w+x \notin S$ when $i \leq r$. Note that $(q-2) x+i+x=(q-1) x+i \leq$ $(q-1) x+r=a-1$. Thus, $(w+x) \bmod a=(q-1) x+i$ and $\left\lfloor\frac{w+x}{a}\right\rfloor x=(q-1) x$, which by Corollary 2 implies that $w+x \notin S$. In addition, let us prove that if $w \in\{q a+(q-1) x+1, \ldots, q a+(q-$ 1) $x+r\}$, then $a$ and $a+x$ are in the same connected component of $\mathrm{G}_{w+(a+x)}$. Take $w=q a+(q-1) x+i$ in this set. Then, $w+(a+x)=$ $q(a+x)+(a+i)$, which means that $[a+x, a+i] \in \mathrm{E}\left(\mathrm{G}_{n}\right)$. Besides, $w+(a+x)=(q+1) a+q x+i=(q+1) a+((q-1) x+r)+x-r+i=$ $(q+1) a+(a-1)+x-r+i=q a+(a+i)+(a+(x-r-1))$ (observe 
that $0 \leq x-r-1 \leq x)$. This implies that $[a, a+i] \in \mathrm{E}\left(\mathrm{G}_{n}\right)$ and consequently $a$ and $a+x$ are connected in $\mathrm{G}_{n}$.

Hence, the elements $w$ we are interested in must be in the set $R=$ $\{(q-1) a+(q-2) x+r+1, \ldots,(q-1) a+(q-2) x+x\}$. Let us show that, as a matter of fact, for the elements $n \in S$ of the form $n=w+(a+x)$, with $w$ in the previous set, the graph $\mathrm{G}_{w+(a+x)}$ has no path connecting $a$ and $a+x$. In order to show this, it is enough to prove that if $w=(q-1) a+(q-2) x+r+i \in R$, $n-((a+k)+(a+j)) \in S$ and $j \geq i$ then $k$ must be greater or equal than $i$. The element $m=n-(a+k+a+j)$ is equal to $(q-2) a+(q-1) x+r+i-k-j$. We distinguish two cases depending on the value of $q-1$ :

- If $q-1 \geq 2$, then $(q-1) x+r+i \geq 2 x \geq k+j$ and therefore $(q-1) x+r+i-k-j \geq 0$. Besides, $(q-1) x+r+i-$ $k-j=(a-1)+i-k-j \leq a-1$ since $i-j \leq 0$. Hence, $m \bmod a=(q-1) x+r+i-k-j$ and $\left\lfloor\frac{m}{a}\right\rfloor x=(q-2) x$. Using Corollary 2, we get that since $m=n-(a+j+a+k) \in S$, $(q-1) x+r+i-j-k \leq(q-2) x$, that is to say, $x+r+i-k-j \leq 0$, and this occurs if and only if $(x-j)+r+i \leq k$ which implies that $k \geq i$, since $x-j+r \geq 0$.

- If $q-1=1$ then $m=x+r+i-k-j \geq 0$, since $m \in S$ and as before $x+r+i-k-j \leq a-1$. In this case, $m \bmod a=$ $x+r+i-k-j$ and $\left\lfloor\frac{m}{a}\right\rfloor=0$. Thus, $m \in S$ implies that $x+r+i-k-j \leq 0$, and this leads to $k \geq i$.

Taking all this into account we have as many new elements in $\rho$ as elements has the set $R$, and this amount is $x-r=x-((a-$ 1) $\bmod x)$.

2) If $r=0$, then $q=\left\lfloor\frac{a-1}{x}\right\rfloor \geq 1$. Let us show that the element $w=k a+(k-1) x+i \in \mathrm{S}(a)(1 \leq i \leq x$ and $0 \leq(k-1) x+i<a)$ satisfies that $w+x$ is not in $S$ when $k \leq q-1$. This is due to the fact that $(k-1) x+i+x=k x+i \leq(q-1) x+i=a-1-x+i<a$ (recall that $i \leq x$ ), and therefore $(w+x) \bmod a=k x+i$ and $\left\lfloor\frac{w+x}{a}\right\rfloor x=k x$, which, once more, implies that $w+x \notin S$.

In this case, we get that $w$ must be in the set $R=\{q a+(q-1) x+$ $1, \ldots, q a+(q-1) x+x\}$. In the same way we did for the case $r \neq 0$, it can be shown that all these elements produce graphs such that $a$ and $a+x$ are in different connected components (the proof is the same but for the cases to distinguish, which are $q-1 \geq 1$ and $q-1=0)$. Again, we get $x=x-0=x-((a-1) \bmod x)$ new elements in $\rho$. 
Counting all the elements in $\rho$, we have that

$$
\# \rho=\frac{x(x-1)}{2}+x-((a-1) \bmod x) .
$$

With this theorem it is easy to prove the next result.

Corollary 9. Let $S=\langle a, a+1, \ldots, a+x\rangle$ be a numerical semigroup with $1 \leq x<a$ and $\rho$ as before. Then, $S$ is a complete intersection if and only if one of the following cases occur:

1) $S=\langle a, a+1\rangle(x=1)$.

2) $S=\langle 2 k, 2 k+1,2 k+2\rangle(x=2$ and $a \bmod 2=0)$.

Proof. The semigroup $S$ is a complete intersection if and only if $\# \rho=x$. Using previous theorem this occurs if and only if $x(x-1) / 2+x-((a-$ 1) $\bmod x)=x$, and this happens if and only if $x(x-1) / 2=(a-1) \bmod x$. Thus, if $S$ is a complete intersection then $x(x-1) / 2<x$ and this implies that $x<3$, since $x>0$. Hence, two cases may occur:

1) $x=1$. In this case, $S=\langle a, a+1\rangle$. Note that if $S$ is of this form then $S$ is a complete intersection.

2) $x=2$. Under this setting then $S=\langle a, a+1, a+2\rangle$. Besides, if we want $S$ to be a complete intersection then $2(2-1) / 2+2-((a-1) \bmod 2)=2$ and this leads to $a \bmod 2=0$.

Finally, we would like to thank the referee for his/her wise comments and suggestions.

\section{References}

[1] R. Apéry, Sur les branches superlinéaires des courbes algébriques, C. R. Acad. Sci. Paris, 222 (1946).

[2] A. Brauer and J. E. Shockley, On a problem of Frobenius, J. Reine Angew. Math., 211 (1962), 215-220.

[3] H. Bresinski, Symmetric semigroups generated by 4 elements, Manuscripta Math., 17 (1995), 81-95.

[4] R. Fröberg, The Frobenius number of some semigroups, Communications in Algebra, 22(14) (1994), 6021-6024.

[5] R. Fröberg, C. Gottlieb and R. Häggkvist, On numerical semigroups, Semigroup Forum, 35 (1987), 63-83.

[6] J. Herzog, Generators and relations of abelian semigroups and semigroup rings, Manuscripta Math., 3 (1970), 175-193.

[7] E. Kunz, The value-semigroup of a one-dimensional Gorenstein ring, Proc. Amer. Math. Soc., 25 (1973), 748-751. 
[8] J.C. Rosales, An algorithmic method to compute a minimal relation for any numerical semigroup, Inter. J. Algebra and Computation, 6(4) (1996), 441-455.

Received January 6, 1998 and revised October 1, 1998. This paper was supported by the project DGES PB96-1424.

UNIVERSIDAD DE GRANADA

Departamento de Álgebra

E-18071 GRANADA

SPAIN

E-mail address: pedro@ugr.es

UNIVERSIDAD DE GRANADA

Departamento de Álgebra

E-18071 GRANADA

SPAIN

E-mail address: jrosales@ugr.es 